\title{
Neue und interessante Milben aus dem Genfer Museum XXXII. Zwei neue Milbenarten (Acari: Acaridae) aus einer Höhle in Kenya
}

von

\author{
S. MAHUNKA*
}

\begin{abstract}
New and interesting mites from the Museum of Geneva XXXII. Two new species of mites (Acari: Acaridae) from a Kenyan cave.
\end{abstract}

\section{Summary}

From a sample of Acaridae mites collected in a Kenyan cave, two new species are described. For one of them a new genus is erected.

Herr Dr. V. Aellen, Direktor des Naturhistorischen Museums in Genf, und Herr Dr. P. Strinati haben in mehreren Höhlen von Kenya zoologische Aufsammlungen durchgeführt. In dem von ihnen gesammelten Milbenmaterial konnte ich - durch die freundliche Vermittlung von Herrn Dr. B. Hauser (Konservator am Naturhistorischen Museum in Genf) - Deutonymphen von Acaridae untersuchen.

Die vorgefundenen Hypopi gehören zu zwei Arten, die beide neu für die Wissenschaft sind. Für eine von ihnen müssen wir eine neue Gattung aufstellen.

Dieses Material verdient deshalb besonderes Interesse, weil aus Afrika bisher keine Höhlenmilben bekannt waren, im Gegensatz zur Höhlenfauna Europas, in der Milben eine ziemliche Bedeutung haben, da bisher eine Reihe von troglobionten Arten entdeckt wurden.

Von den parasitischen Arten abgesehen, ernährt sich ein Teil der Höhlenmilben von dem häufig reichlich vorhandenen Guano, ein anderer Teil dagegen von eingeschwemmten, organischen Stoffen, wie Mulm etc. Die Imagines der beiden neuen Arten gehören ohne Zweifel zu der letzteren Gruppe.

* Dr. Sándor Mahunka, Zoologische Abteilung des Üngarischen Naturwissenschaftlichen Museums, Baross ucta 13, H-1088 Budapest, Ungarn. 
Aellenella gen. nov.

Diagnose: Hypopus. Körper regelmäßig entwickelt. Die 1. und 2. Beinpaare sind wesentlich größer (länger und breiter) als die 3. und 4. Auf sämtlichen Beinen sind lanzettförmige Haare vorhanden. Auf dem Tarsus des 1. Beines entspringen die $\omega_{1}, \omega_{2}$ und $\omega_{\mathrm{x}}$ Solenidien. Auf der Tibia des 1. und 2. Beines steht nur 1-1 Haar, das Haar 1" fehlt.

Typus-Art: Aellenella strinatii sp. nov.

Bemerkung: Die Systematik der Überfamilie Acaroidea konnte bisher wegen der noch immer mangelhaft erforschten Imagines bei weitem nicht aufgeklärt werden, denn allein auf Grund der Hypopen lassen sich die verwandtschaftlichen Beziehungen nicht klarlegen. Die oben beschriebene Deutonymphe läßt sich aber auf Grund der Morphologie und Behaarung der Gliedmaßen mit großer Sicherheit in das System der Familie Acaridae einordnen und zwar in den Verwandtschaftskreis der Unterfamilie Rhyzoglyphinae. Doch auf Grund der Solenidiengruppe des 1 . Beines und des fehlenenden 2. Haares (1") der 1 . und 2. Tibia kann man diese Form von allen bis heute bekannten AcaridaeHypopi sofort unterscheiden.

Diese neue Gattung widme ich Herrn Direktor Dr. V. Aellen als Ausdruck meiner Dankbarkeit.

Aellenella strinatii sp. nov.

Dimensionen. - Länge: 170-176 $\mu$, Breite: 101-104 $\mu$.

Habitus: Propodosoma nach vorne sich verschmälernd, Hysterosoma parallelseitig, nur hinten abgerundet. Dorsalfläche des Propodosoma und Hysterosoma stark punktiert. Dorsosejugalregion fein gestreift. Farbe weißlich gelb.

Dorsalansicht (Abb. 1): Auf dem Propodosoma entspringende Haare sind gleich lang und stehen in einer Querlinie. Auf dem Propodosoma stehen 10 Haarpaare, von denen die vorne stehenden ganz kurz sind, die hinten stehenden länger, besonders lang die Haare $h_{1}$.

Ventralansicht (Abb. 4): Vor dem Gnathosoma, auf einer eckigen Querlinie entspringen zwei ziemlich starke Haare. Auf der Coxisternalregion sind die A podemen gut entwickelt, das ap. sa. endet frei, die ap. 2. bilden gebogene Epimeralflecke. Die Sejugalapodemen verschmelzen. Die ap. sp., ap. 3, ap. 4 bilden ein geschlossenes Netz. Auf den 1., 3. und 4. Epimeren je ein ganz schwach entwickelter Saugnapf; neben der Kreuzung der 4. und hinteren Sternalapodemen, in der Mitte und neben der Genitalöffnung je auch ein Haarpaar. Saugnapfplatte groß, steht ganz hinten, nahe dem Körperende. Die DsNäpfe ganz schwach entwickelt.

Gnathosoma (Abb. 3): Das Infracapitulum fassförmig, die Palpen sind ganz kurz. Die Solenidien und auch die Haare sind lang.

Abb. 1-8. Aellenella strinatii gen. n., sp. n. - 1: Dorsalansicht, 2: Bein 3, 3: Gnathosoma, 4: Ventralansicht, 5: Bein 1, 6: Bein 1 vom Ventral, 7: Bein 4,8: Bein 2. 


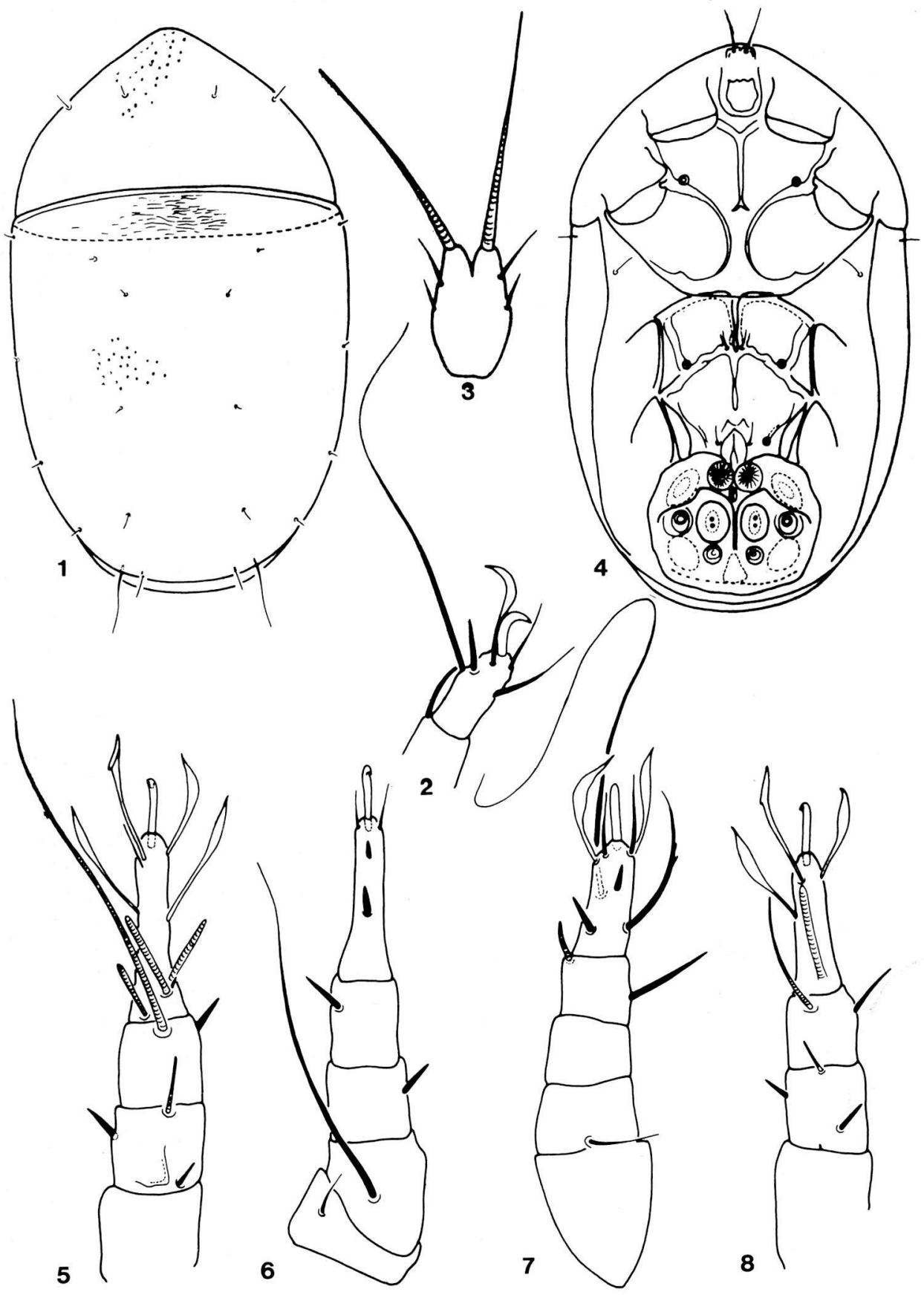


Beine: Auf der allen Beinen sitzen gleichgroße und gleichförmige Krallen. Auf dem Tarsus des 1. Beines (Abb. 5) entspringen 3 lanzettförmige Haare. Alle Solenidien stehen im Basalteil des Tarsus. Auf der Tibia entspringt nur 1 Haar, das Haar 1" fehlt. 'Auf der Ventralseite des Femurs des 1. (Abb. 6) und 2. Beines (Abb. 8) ein sehr langes Haar, es ist fast so lang wie die Länge des Beines, Auf dem 3. und 4. Bein (Abb. 2,7) gibt es 2-2 lanzettförmige Haare und 1-1 langes Schlepphaar.

Untersuchungsmaterial: Holotypus: Kenya, grotte de Similani $10 \mathrm{~km}$. S Mombasa. 29.IX.1975. leg. P. Strinati et V. Aellen; I Paratyp vom selben Fundort. Der Holotypus in der Sammlung des Naturhistorisches Museums Genf, der Paratypus in der Sammlung des Naturhistorisches Museums Budapest unter der Inventarnummer (103-PA-76) deponiert.

Bemerkung: Wie bei der Diagnose der Gattung erwähnt, ist die neue Art alleinstehend zwischen den bisher bekannten Acaridae-Arten.

Die neue Art benenne ich zu Ehren von Herrn Dr. P. Strinati, dem bekannten Biospeläologen und Forschungsreisenden.

\section{Schwiebea cavicola sp. nov.}

\section{Dimensionen: Länge: 173-311 $\mu$, Breite: 148-247 $\mu$.}

Habitus: Der Körper fast regelmäßig ellipsförmig, hinten abgerundet; vorne bei einigen Exemplaren das Propodosoma ein wenig zugespitzt, und in Mitte der Seite des Propodosomas noch eine schwache, dreieckige Erhöhung. Die Oberfläche des Körpers mit sehr feinen, aber dicht nebeneinander stehenden Punkten ornamentiert.

Dorsalansicht (Abb. 9): Von den auf dem Propodosoma entspringenden Haaren steht das innere Paar vor dem äußeren und ist etwas kürzer. Die Dorsosejugalregion mit feinen, aufgeteilten Streifen. Auf dem Hysterosoma entspringen 10 Paar Haare, das Haar $h_{1}$ länger als die übrigen.

Ventralansicht (Abb. 12): Die Apodemen schwach entwickelt, vorderes Sternalapodema endet frei, die 2 Apodemen setzen sich in gebogenen dünnen Linien in der Richtung der Sejugalapodemen fort. Auf der hinteren Sternalplatte ist kein hinteres Sternalapodema entwickelt. Zwischen den 3. und 4. Epimeren befindet sich ein ziemlich breites Feld. Die Haare des 3. Epimeren stehen weit voneinander. Auf den 1., 3. und 4. Epimeren gibt es je einen Saugnapf. Die Saugnapfplatte befindet sich weit vom Hinterrand des Körpers, hinter dieser sieht man 4 breite, fingerförmige Ausbildungen.

Gnathosoma (Abb. 10): Vorne sich schwach verschmälernd, die Palpen gut sichtbar. Die Haare des Infracapitulum etwas kürzer als die auf Palpen entspringenden.

Beine: Auf dem Tarsus des 1. und 2. Beines (Abb. 13, 14, 15) befinden sich 5 lanzettförmige Haare. Die $\omega_{1}$ Solenidien sind sehr lang und dick, ihre distalen Ende keulenförmig verdickt. Die $\varphi_{1}$ Solenidien des 3. und 4. Beines (Abb. 11 , 15) sehr lang, die $\varphi_{1}$ des 3 . Beines reichen weit über das Ende des Tarsus. Auf beiden Beinen entspringen 5-5 lanzettförmige Haare, ihre Form und Größe sind verschieden. 

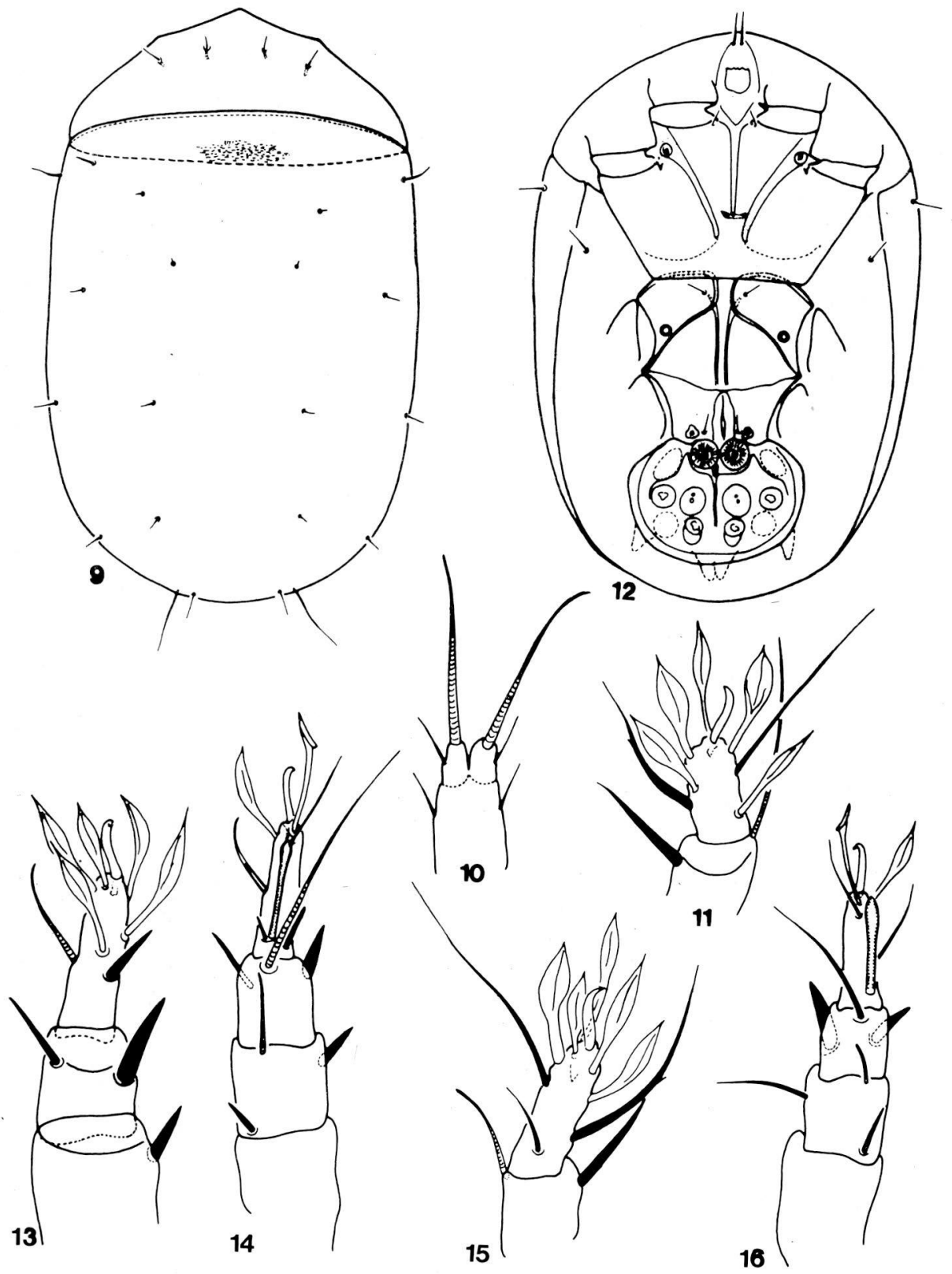

Abb. 9-16. Schwiebea cavicola sp. n. - 9: Dorsalansicht, 10: Gnathosoma, 11: Bein 3, 12: Ventralansicht, 13: Bein 1 von Ventral, 14: Bein 1, 15: Bein 4, 16: Bein 2. 
Untersuchungsmaterial: Holotype: Kenya, grotte de Similani $10 \mathrm{~km}$. S Mombasa, 29.IX.1975. leg. P. Strinati et V. Aellen; 5 Paratypen: mit dem Holotypus gesammelt. Der Holotypus und 3 Paratypen befinden sich in der Sammlung des Naturhistorischen Museums Genf, 2 Paratypen, unter der Inventarnummer 104-PA-76, in der Sammlung des Naturhistorischen Museums Budapest aufbewahrt.

Bemerkung: Nur der Hypopus bekannt. Auf Grund der lanzettförmigen Haare der Beine und der Ausbildung der Apodemen gehört die neue Art wahrscheinlich zu der Gattung Schwiebea Oudemans, 1916. Von der bisher bekannten Schwiebea-Arten unterscheidet sie sich durch die stark punktierte Dorsalfläche des Körpers, die Form des Sensillus, des 1. und 2. Beines und durch die fingerförmige Ausbildung hinter der Saugnapfplatte.

Diese Gattung erweist sich als äußerst heterogen, doch kann sie - wie weiter oben schon dargelegt wurde - nur nach Erforschung der Imagines in kleinere Gruppen aufgeteilt werden. Aus Afrika kennen wir schon oehrere Arten, die als zu dieser Gattung gehörende Formen beschrieben worden sind, ihre Mehrzahl gehört aber gewiss nicht zu dieser Gattung. Auch aus Höhlen sind schon einige Arten dieser Gattung bekannt geworden (z.B. Schwiebea cavernicola Vitzthum, 1922 ist sicherlich troglobiont).

\section{ZUSAMMENFASSUNG}

Aus einem in einer Höhle von Kenya gesammelten Milbenmaterial wurden die Acaridae-Arten untersucht. Beide vorkommenden Arten waren neu für die Wissenschaft, für eine mußte auch eine neue Gattung aufgestellt werden.

\section{LITERATUR}

MAHUNKA, S. 1977: XX. Contribution to the Oribatid Fauna of SE Asia (Acari, Oribatida). Revue suisse Zool. 84, 247-274.

TÜRK, E. und TÜRK, F. (1957): Systematik und Ökologie der Tyroglyphiden Mitteleuropas. in: Stammer, H. J.: Beiträge zur Syst., Ökol. Mitteleuropäischer Acarina, 1, 1: 1-231.

ZACHVATKIN, A. A. (1941): Tyroglyphoidea. - in: Fauna SSSR, Arachnoidea 6: 1-573. 